\title{
A New Iterative Method for Equilibrium Problems and Fixed Point Problems
}

\author{
Abdul Latif ${ }^{1}$ and Mohammad Eslamian ${ }^{2}$ \\ ${ }^{1}$ Department of Mathematics, King Abdulaziz University, P.O. Box 80203, Jeddah 21589, Saudi Arabia \\ ${ }^{2}$ Department of Mathematics, Mazandaran University of Science and Technology, Behshahr, Iran
}

Correspondence should be addressed to Abdul Latif; alatif@kau.edu.sa

Received 31 October 2013; Accepted 15 December 2013

Academic Editor: Ljubomir B. Ćirić

Copyright (C) 2013 A. Latif and M. Eslamian. This is an open access article distributed under the Creative Commons Attribution License, which permits unrestricted use, distribution, and reproduction in any medium, provided the original work is properly cited.

Introducing a new iterative method, we study the existence of a common element of the set of solutions of equilibrium problems for a family of monotone, Lipschitz-type continuous mappings and the sets of fixed points of two nonexpansive semigroups in a real Hilbert space. We establish strong convergence theorems of the new iterative method for the solution of the variational inequality problem which is the optimality condition for the minimization problem. Our results improve and generalize the corresponding recent results of Anh (2012), Cianciaruso et al. (2010), and many others.

\section{"Dedicated to Professor Miodrag Mateljevic on the occasion of his 65th birthday"}

\section{Introduction}

Let $H$ be a real Hilbert space with inner product $\langle\cdot, \cdot\rangle$ and norm $\|\cdot\|$. Let $C$ be a nonempty closed convex subset of $H$, and let Proj ${ }_{C}$ be a nearest point projection of $H$ into $C$; that is, for $x \in H, \operatorname{Proj}_{C} x$ is the unique point in $C$ with the property $\left\|x-\operatorname{Proj}_{C} x\right\|:=\inf \{\|x-y\|: y \in C\}$. It is well known that $y=\operatorname{Proj}_{C} x$ iff $\langle x-y, y-z\rangle \geq 0$ for all $z \in C$.

Let $f$ be a bifunction from $C \times C$ into $\mathbb{R}$, such that $f(x, x)=0$ for all $x \in C$. Consider the Fan inequality [1]: find a point $x^{\star} \in C$ such that

$$
f\left(x^{\star}, y\right) \geq 0, \quad \forall y \in C,
$$

where $f(x, \cdot)$ is convex and subdifferentiable on $C$ for every $x \in C$. The set of solutions of this problem is denoted by $\operatorname{Sol}(f, C)$. In fact, the Fan inequality can be formulated as an equilibrium problem. Such problems arise frequently in mathematics, physics, engineering, game theory, transportation, economics, and network. Due to importance of the solutions of such problems, many researchers are working in this area and studying the existence of the solutions of such problems; for example, see, [2-4]. Further, if $f(x, y)=$ $\langle F x, y-x\rangle$ for every $x, y \in C$, where $F$ is a mapping from $C$ into $H$, then the Fan inequality problem (equilibrium problem) becomes the classical variational inequality problem which is formulated as finding a point $x^{\star} \in C$ such that

$$
\left\langle F x^{\star}, y-x^{\star}\right\rangle \geq \quad \forall y \in C .
$$

Variational inequalities were introduced and studied by Stampacchia [5]. It is well known that this area covers many branches of mathematics, such as partial differential equations, optimal control, optimization, mathematical programming, mechanics, and finance; see [6-11].

Here we recall some useful notions.

A mapping $T: C \rightarrow H$ is said to be L-Lipschitz on $C$ if there exists a constant $L \geq 0$ such that for each $x, y \in C$,

$$
\|T x-T y\| \leq L\|x-y\| .
$$

In particular, if $L \in[0,1[$, then $T$ is called a contraction on $C$; if $L=1$, then $T$ is called a nonexpansive mapping on $C$. The set of fixed points of $T$ is denoted by $F(T)$.

A family $\mathscr{T}:=\{T(s): 0 \leq s<\infty\}$ of mappings on a closed convex subset $C$ of a Hilbert space $H$ is called a nonexpansive semigroup if it satisfies the following: (i) $T(0) x=x$ for all $x \in C$; (ii) $T(s+t)=T(s) T(t)$ for all $s, t \geq 0$; (iii) for all $x \in C$, 
$s \rightarrow T(s) x$ is continuous; (iv) $\|T(s) x-T(s) y\| \leq\|x-y\|$ for all $x, y \in C$ and $s \geq 0$.

We use $F(\mathscr{T})$ to denote the common fixed point set of the semigroup $\mathscr{T}$; that is, $F(\mathscr{T})=\{x \in C: T(s) x=x, \forall s \geq$ $0\}$. It is well known that $F(\mathscr{T})$ is closed and convex [12]. A nonexpansive semigroup $\mathscr{T}$ on $C$ is said to be uniformly asymptotically regular (in short, u.a.r.) on $C$ if for all $h \geq 0$ and any bounded subset $B$ of $C$,

$$
\lim _{t \rightarrow \infty} \sup _{x \in B}\|T(h)(T(t) x)-T(t) x\|=0
$$

For each $h \geq 0$, define $\sigma_{t}(x)=(1 / t) \int_{0}^{t} T(s) x d s$. Then

$$
\lim _{t \rightarrow \infty} \sup _{x \in B}\left\|T(h)\left(\sigma_{t}(x)\right)-\sigma_{t}(x)\right\|=0,
$$

Provided that $B$ is closed bounded convex subset of $C$. It is known that the set $\left\{\sigma_{t}(x): t>0\right\}$ is a u.a.r. nonexpansive semigroup; see [13]. The other examples of u.a.r. operator semigroup can be found in [14].

A bifunction $f: C \times C \rightarrow \mathbb{R}$ is said to be (i) strongly monotone on $C$ with $\alpha>0$ if $f(x, y)+f(y, x) \leq-\alpha\|x-y\|^{2}$, $\forall x, y \in C$; (ii) monotone on $C$ if $f(x, y)+f(y, x) \leq 0, \forall x, y \in$ $C$; (iii) pseudomonotone on $C$ if $f(x, y) \geq 0 \Rightarrow f(y, x) \leq 0$, $\forall x, y \in C$; (iv) Lipschitz-type continuous on $C$ with constants $c_{1}>0$ and $c_{2}>0$ if $f(x, y)+f(y, z) \geq f(x, z)-c_{1}\|x-y\|^{2}-$ $c_{2}\|y-z\|^{2}, \forall x, y, z \in C$.

Note that if $T$ is $L$-Lipschitz on $C$, then for each $x, y \in$ $C$, the function $f(x, y)=\langle F x, y-x\rangle$ is a Lipschitz-type continuous with constants $c_{1}=c_{2}=L / 2$.

An operator $A$ on $H$ is called strongly positive if there is a constant $\bar{\gamma}>0$ such that

$$
\langle A x, x\rangle \geq \bar{\gamma}\|x\|^{2}, \quad \forall x \in H
$$

Recently, iterative methods for nonexpansive mappings have been applied to solve convex minimization problems. In [15], Xu defined an iterative sequence $\left\{x_{n}\right\}$ in $C$ which converges strongly to the unique solution of the minimization problem under some suitable conditions. A well-known typical problem is to minimize a quadratic function over the set of fixed points of a nonexpansive mapping $T$ on a real Hilbert space $H$ :

$$
\min _{x \in F(T)} \frac{1}{2}\langle A x, x\rangle-\langle x, b\rangle
$$

where $b$ is a given point in $H$ and $A$ is strongly positive operator.

For solving the variational inequality problem, Marino and $\mathrm{Xu}[16]$ introduced the following general iterative process for nonexpansive mapping $T$ based on the viscosity approximation method (see [17]):

$$
x_{n+1}=a_{n} \gamma h\left(x_{n}\right)+\left(I-a_{n} A\right) T x_{n}, \quad \forall n \geq 0,
$$

where $A$ is strongly positive bounded linear operator on $H$, $h$ is contraction on $H$, and $\left.\left\{a_{n}\right\} \subset\right] 0,1$. They proved that, under some appropriate conditions on the parameters, the sequence $\left\{x_{n}\right\}$ generated by (8) converges strongly to the unique solution $x^{\star} \in F(T)$ of the variational inequality

$$
\left\langle(A-\gamma h) x^{\star}, x-x^{\star}\right\rangle \geq 0, \quad \forall x \in F(T),
$$

which is the optimality condition for the minimization problem

$$
\min _{x \in F(T)} \frac{1}{2}\langle A x, x\rangle-g(x)
$$

where $g$ is a potential function for $\gamma h$ (i.e., $g^{\prime}(x)=\gamma h(x)$, $\forall x \in H)$.

Iterative process for approximating common fixed points of a nonexpansive semigroup has been investigated by various authors (see [13, 14, 18-21]). Recently, Li et al. [19] introduced the following iterative procedure for the approximation of common fixed points of a nonexpansive semigroup $\mathscr{T}=$ $\{T(s): 0 \leq s<\infty\}$ on a closed convex subset $C$ of a Hilbert space $H$ :

$$
x_{n}=\alpha_{n} \gamma h\left(x_{n}\right)+\left(I-\alpha_{n} A\right) \frac{1}{s_{n}} \int_{0}^{s_{n}} T(s) x_{n} d s, \quad n \geq 1,
$$

where $A$ is a strongly positive bounded linear operator on $H$ and $h$ is a contraction on $C$. Imposing some appropriate conditions on the parameters, they proved that the iterative sequence $\left\{x_{n}\right\}$ generated by (11) converges strongly to the unique solution $x^{\star} \in F(\mathscr{T})$ of the variational inequality $\left\langle(\gamma h-A) x^{\star}, z-x^{\star}\right\rangle \leq 0, \forall z \in F(\mathscr{T})$.

For obtaining a common element of $\operatorname{Sol}(f, C)$ and the set of fixed points of a nonexpansive mapping $T$, S. Takahashi and W. Takahashi [9] first introduced an iterative scheme by the viscosity approximation method. They proved that under certain conditions the iterative sequences converge strongly to $z=\operatorname{Proj}_{F(T) \cap \operatorname{Sol}(f, C)}(h(z))$.

During last few years, iterative algorithms for finding a common element of the set of solutions of Fan inequality and the set of fixed points of nonexpansive mappings in a real Hilbert space have been studied by many authors (see, e.g., [2, 4, 22-28]). Recently, Anh [22] studied the existence of a common element of the set of fixed points of a nonexpansive mapping and the set of solutions of Fan inequality for monotone and Lipschitz-type continuous bifunctions. He introduced the following new iterative process:

$$
\begin{array}{r}
w_{n}=\operatorname{argmin}\left\{\lambda_{n} f\left(x_{n}, w\right)+\frac{1}{2}\left\|w-x_{n}\right\|^{2}: w \in C\right\}, \\
z_{n}=\operatorname{argmin}\left\{\lambda_{n} f\left(w_{n}, z\right)+\frac{1}{2}\left\|z-x_{n}\right\|^{2}: z \in C\right\}, \\
x_{n+1}=\alpha_{n} h\left(x_{n}\right)+\beta_{n} x_{n}+\gamma_{n}\left(\mu S\left(x_{n}\right)+(1-\mu) z_{n}\right), \\
\forall n \geq 0,
\end{array}
$$

where $\mu \in] 0,1[, C$ is nonempty, closed convex subset of a real Hilbert space $H, f$ is monotone, continuous, and Lipschitztype continuous bifunction, $h$ is self-contraction on $C$ with constant $k \in] 0,1$ [, and $S$ is self nonexpansive mapping on $C$. He proved that, under some appropriate conditions over 
positive sequences $\left\{\alpha_{n}\right\},\left\{\beta_{n}\right\},\left\{\gamma_{n}\right\}$, and $\left\{\lambda_{n}\right\}$, the sequences $\left\{x_{n}\right\},\left\{w_{n}\right\}$, and $\left\{z_{n}\right\}$ converge strongly to $q \in F(S) \cap \operatorname{Sol}(f, C)$ which is a solution of the variational inequality $\langle(I-h) q, x-$ q) $\geq 0, \forall x \in F(S) \cap \operatorname{Sol}(f, C)$.

In this paper, we introduce a new iterative scheme based on the viscosity method and study the existence of a common element of the set of solutions of equilibrium problems for a family of monotone, Lipschitz-type continuous mappings and the sets of fixed points of two nonexpansive semigroups in a real Hilbert space. We establish strong convergence theorems of the new iterative scheme for the solution of the variational inequality problem which is the optimality condition for the minimization problem. Our results improve and generalize the corresponding recent results of Anh [22], Cianciaruso et al. [18], and many others.

\section{Preliminaries}

In this section we collect some lemmas which are crucial for the proofs of our results.

Let $\left\{x_{n}\right\}$ be a sequence in $H$ and $x \in H$. In the sequel, $x_{n} \rightarrow x$ denotes that $\left\{x_{n}\right\}$ weakly converges to $x$ and $x_{n} \rightarrow x$ denotes that $\left\{x_{n}\right\}$ weakly converges to $x$.

Lemma 1. Let $H$ be a real Hilbert space. Then the following inequality holds:

$$
\|x+y\|^{2} \leq\|x\|^{2}+2\langle y, x+y\rangle, \quad \forall x, y \in H .
$$

Lemma 2 (see [15]). Assume that $\left\{a_{n}\right\}$ is a sequence of nonnegative real numbers such that

$$
a_{n+1} \leq\left(1-\eta_{n}\right) a_{n}+\eta_{n} \delta_{n}, \quad n \geq 0,
$$

where $\left\{\eta_{n}\right\}$ is a sequence in $] 0,1\left[\right.$ and $\delta_{n}$ is a sequence in $\mathbb{R}$ such that

(i) $\sum_{n=1}^{\infty} \eta_{n}=\infty$,

(ii) $\lim \sup _{n \rightarrow \infty} \delta_{n} \leq 0$ or $\sum_{n=1}^{\infty}\left|\eta_{n} \delta_{n}\right|<\infty$.

Then $\lim _{n \rightarrow \infty} a_{n}=0$.

Lemma 3 (see [16]). Let $A$ be a strongly positive linear bounded self-adjoint operator on $H$ with coefficient $\bar{\gamma}>0$ and $0<\rho \leq\|A\|^{-1}$. Then $\|I-\rho A\| \leq 1-\rho \bar{\gamma}$.

Lemma 4 (see [29]). Let $H$ be a Hilbert space and $x_{i} \in H$, $(1 \leq i \leq m)$. Then for any given $\left.\left\{\lambda_{i}\right\}_{i=1}^{m} \subset\right] 0,1\left[\right.$ with $\sum_{i=1}^{m} \lambda_{i}=$ 1 and for any positive integer $k, j$ with $1 \leq k<j \leq m$,

$$
\left\|\sum_{i=1}^{m} \lambda_{i} x_{i}\right\|^{2} \leq \sum_{i=1}^{m} \lambda_{i}\left\|x_{i}\right\|^{2}-\lambda_{k} \lambda_{j}\left\|x_{k}-x_{j}\right\|^{2} .
$$

Lemma 5 (see [30]). Let $\left\{t_{n}\right\}$ be a sequence of real numbers such that there exists a subsequence $\left\{n_{i}\right\}$ of $\{n\}$ such that $t_{n_{i}}<$ $t_{n_{i}+1}$ for all $i \in \mathbb{N}$. Then there exists a nondecreasing sequence $\{\tau(n)\} \subset \mathbb{N}$ such that $\tau(n) \rightarrow \infty$ and the following properties are satisfied by all (sufficiently large) numbers $n \in \mathbb{N}$ :

$$
t_{\tau(n)} \leq t_{\tau(n)+1}, \quad t_{n} \leq t_{\tau(n)+1} .
$$

In fact

$$
\tau(n)=\max \left\{k \leq n: t_{k}<t_{k+1}\right\} .
$$

Lemma 6 (see [2]). Let $C$ be a nonempty closed convex subset of a real Hilbert space $H$ and let $f: C \times C \rightarrow \mathbb{R}$ be a pseudomonotone and Lipschitz-type continuous bifunction with constants $c_{1}, c_{2} \geq 0$. For each $x \in C$, let $f(x, \cdot)$ be convex and subdifferentiable on $C$. Let $x_{0} \in C$ and let $\left\{x_{n}\right\},\left\{z_{n}\right\}$, and $\left\{w_{n}\right\}$ be sequences generated by

$$
\begin{aligned}
& w_{n}=\operatorname{argmin}\left\{\lambda_{n} f\left(x_{n}, w\right)+\frac{1}{2}\left\|w-x_{n}\right\|^{2}: w \in C\right\}, \\
& z_{n}=\operatorname{argmin}\left\{\lambda_{n} f\left(w_{n}, z\right)+\frac{1}{2}\left\|z-x_{n}\right\|^{2}: z \in C\right\} .
\end{aligned}
$$

Then for each $x^{\star} \in \operatorname{Sol}(f, C)$,

$$
\begin{aligned}
\left\|z_{n}-x^{\star}\right\|^{2} \leq & \left\|x_{n}-x^{\star}\right\|^{2}-\left(1-2 \lambda_{n} c_{1}\right)\left\|x_{n}-w_{n}\right\|^{2} \\
& -\left(1-2 \lambda_{n} c_{2}\right)\left\|w_{n}-z_{n}\right\|^{2}, \quad \forall n \geq 0 .
\end{aligned}
$$

\section{Main Results}

In this section, we prove the main strong convergence result which solves the problem of finding a common element of three sets $F(\mathscr{T}), F(\mathcal{S})$, and $\operatorname{Sol}\left(f_{i}, C\right)$ for finite family of monotone, continuous, and Lipschitz-type continuous bifunctions $f_{i}$ in a real Hilbert space $H$.

Theorem 7. Let $C$ be a nonempty closed convex subset of a real Hilbert space $H$ and let $f_{i}: C \times C \rightarrow \mathbb{R}(i=1,2, \ldots, m)$ be a finite family of monotone, continuous, and Lipschitztype continuous bifunctions with constants $c_{1, i}$ and $c_{2, i}$. Let $\mathscr{T}=\{T(u): u \geq 0\}$ and $\mathcal{S}=\{S(u): u \geq 0\}$ be two u.a.r. nonexpansive self-mapping semigroups on $C$ such that $\Omega=\bigcap_{i=1}^{m} \operatorname{Sol}\left(f_{i}, C\right) \bigcap F(\mathscr{T}) \bigcap F(\mathcal{S}) \neq \emptyset$. Assume that $h$ is a $k$-contraction self-mapping of $C$ and $A$ is a strongly positive bounded linear self-adjoint operator on $H$ with coefficient $\bar{\gamma}<$ 1 and $0<\gamma<\bar{\gamma} / k$. Let $x_{0} \in C$ and let $\left\{x_{n}\right\},\left\{w_{n, i}\right\}$, and $\left\{z_{n, i}\right\}$ be sequences generated by

$$
\begin{array}{r}
w_{n, i}=\operatorname{argmin}\left\{\lambda_{n, i} f_{i}\left(x_{n}, w\right)+\frac{1}{2}\left\|w-x_{n}\right\|^{2}: w \in C\right\}, \\
i \in\{1,2, \ldots, m\}, \\
z_{n, i}=\operatorname{argmin}\left\{\lambda_{n, i} f_{i}\left(w_{n, i}, z\right)+\frac{1}{2}\left\|z-x_{n}\right\|^{2}: z \in C\right\}, \\
i \in\{1,2, \ldots, m\}, \\
y_{n}=\alpha_{n} x_{n}+\sum_{i=1}^{m} \beta_{n, i} z_{n, i}+\gamma_{n} T\left(t_{n}\right) x_{n}+\eta_{n} S\left(t_{n}\right) x_{n}, \\
x_{n+1}=\theta_{n} \gamma h\left(x_{n}\right)+\left(I-\theta_{n} A\right) y_{n}, \quad \forall n \geq 0,
\end{array}
$$


where $\alpha_{n}+\sum_{i=1}^{m} \beta_{n, i}+\gamma_{n}+\eta_{n}=1$ and $\left\{t_{n}\right\},\left\{\alpha_{n}\right\},\left\{\beta_{n, i}\right\},\left\{\gamma_{n}\right\}$, $\left\{\eta_{n}\right\},\left\{\lambda_{n, i}\right\}$, and $\left\{\theta_{n}\right\}$ satisfy the following conditions:

(i) $\lim _{n \rightarrow \infty} t_{n}=\infty$,

(ii) $\left.\left\{\theta_{n}\right\} \subset\right] 0,1\left[, \lim _{n \rightarrow \infty} \theta_{n}=0\right.$, and $\sum_{n=1}^{\infty} \theta_{n}=\infty$,

(iii) $\left.\left\{\lambda_{n, i}\right\} \subset[a, b] \subset\right] 0,1 / L\left[\right.$, where $L=\max \left\{2 c_{1, i}, 2 c_{2, i}, 1 \leq\right.$ $i \leq m\}$,

(iv) $\left.\left\{\alpha_{n}\right\},\left\{\beta_{n, i}\right\},\left\{\gamma_{n}\right\},\left\{\eta_{n}\right\} \subset\right] 0,1\left[, \liminf _{n} \alpha_{n} \beta_{n, i}>0\right.$, $\liminf _{n} \alpha_{n} \gamma_{n}>0, \liminf _{n} \alpha_{n} \eta_{n}>0$, and $\lim \inf _{n} \beta_{n, i}\left(1-2 \lambda_{n, i} c_{1, i}\right)>0$ for each $1 \leq i \leq m$.

Then, the sequence $\left\{x_{n}\right\}$ converges strongly to $x^{\star} \in$ $\bigcap_{i=1}^{m} \operatorname{Sol}\left(f_{i}, C\right) \bigcap F(\mathscr{T}) \bigcap F(\mathcal{S})$ which solves the variational inequality:

$$
\left\langle(A-\gamma h) x^{\star}, x-x^{\star}\right\rangle \geq 0, \quad \forall x \in \Omega .
$$

Proof. Since $F(\mathscr{T}), F(\mathcal{S})$, and $\operatorname{Sol}\left(f_{i}, C\right)$ are closed and convex, $\operatorname{Proj}_{\Omega}$ is well-defined. We claim that $\operatorname{Proj}_{\Omega}(I-A+\gamma h)$ is a contraction from $C$ into itself. Indeed, for each $x, y \in C$, we have

$$
\begin{aligned}
& \left\|\operatorname{Proj}_{\Omega}(I-A+\gamma h)(x)-\operatorname{Proj}_{\Omega}(I-A+\gamma h)(y)\right\| \\
& \quad \leq\|(I-A+\gamma h)(x)-(I-A+\gamma h)(y)\| \\
& \quad \leq\|(I-A) x-(I-A) y\|+\gamma\|h x-h y\| \\
& \quad \leq(1-\bar{\gamma})\|x-y\|+\gamma k\|x-y\| \\
& \quad \leq(1-(\bar{\gamma}-\gamma k))\|x-y\| .
\end{aligned}
$$

Therefore, by the Banach contraction principle, there exists a unique element $x^{\star} \in C$ such that $x^{\star}=\operatorname{Proj}_{\Omega}(I-A+$ $\gamma h) x^{\star}$. We show that $\left\{x_{n}\right\}$ is bounded. Since $\lim _{n \rightarrow \infty} \theta_{n}=0$, we can assume, with no loss of generality, that $\theta_{n} \in\left(0,\|A\|^{-1}\right)$, for all $n \geq 0$. By Lemma 6 , for each $1 \leq i \leq m$, we have

$$
\left\|z_{n, i}-x^{\star}\right\| \leq\left\|x_{n}-x^{\star}\right\| .
$$

This implies that

$$
\begin{aligned}
& \left\|y_{n}-x^{\star}\right\| \\
& \leq\left\|\alpha_{n} x_{n}+\sum_{i=1}^{m} \beta_{n, i} z_{n, i}+\gamma_{n} T\left(t_{n}\right) x_{n}+\eta_{n} S\left(t_{n}\right) x_{n}-x^{\star}\right\| \\
& \leq \alpha_{n}\left\|x_{n}-x^{\star}\right\|+\sum_{i=1}^{m} \beta_{n, i}\left\|z_{n, i}-x^{\star}\right\| \\
& \quad+\gamma_{n}\left\|T\left(t_{n}\right) x_{n}-x^{\star}\right\|+\eta_{n}\left\|S\left(t_{n}\right) x_{n}-x^{\star}\right\| \\
& \leq \alpha_{n}\left\|x_{n}-x^{\star}\right\|+\sum_{i=1}^{m} \beta_{n, i}\left\|x_{n}-x^{\star}\right\| \\
& \quad+\gamma_{n}\left\|x_{n}-x^{\star}\right\|+\eta_{n}\left\|x_{n}-x^{\star}\right\| \\
& =\left\|x_{n}-x^{\star}\right\| .
\end{aligned}
$$

It follows from Lemma 3 that

$$
\begin{aligned}
&\left\|x_{n+1}-x^{\star}\right\| \\
&=\left\|\theta_{n}\left(\gamma h\left(x_{n}\right)-A x^{\star}\right)+\left(I-\theta_{n} A\right)\left(y_{n}-x^{\star}\right)\right\| \\
& \leq \theta_{n}\left\|\gamma h\left(x_{n}\right)-A x^{\star}\right\|+\left\|I-\theta_{n} A\right\|\left\|y_{n}-x^{\star}\right\| \\
& \leq \theta_{n}\left\|\gamma h\left(x_{n}\right)-A x^{\star}\right\|+\left(1-\theta_{n} \bar{\gamma}\right)\left\|x_{n}-x^{\star}\right\| \\
& \leq \theta_{n} \gamma\left\|h\left(x_{n}\right)-h\left(x^{\star}\right)\right\|+\theta_{n}\left\|\gamma h\left(x^{\star}\right)-A x^{\star}\right\| \\
&+\left(1-\theta_{n} \bar{\gamma}\right)\left\|x_{n}-x^{\star}\right\| \\
& \leq \theta_{n} \gamma k\left\|x_{n}-x^{\star}\right\|+\theta_{n}\left\|\gamma h\left(x^{\star}\right)-A x^{\star}\right\| \\
&+\left(1-\theta_{n} \bar{\gamma}\right)\left\|x_{n}-x^{\star}\right\| \\
& \leq\left(1-\theta_{n}(\bar{\gamma}-\gamma k)\right)\left\|x_{n}-x^{\star}\right\|+\theta_{n}\left\|\gamma h\left(x^{\star}\right)-A x^{\star}\right\| \\
&=\left(1-\theta_{n}(\bar{\gamma}-\gamma k)\right)\left\|x_{n}-x^{\star}\right\| \\
&+\theta_{n}(\bar{\gamma}-\gamma k) \frac{\left\|\gamma h\left(x^{\star}\right)-A x^{\star}\right\|}{\bar{\gamma}-\gamma k} \\
& \leq \max \left\{\left\|x_{n}-x^{\star}\right\|, \frac{\left\|\gamma h x^{\star}-\mathscr{A} x^{\star}\right\|}{\bar{\gamma}-\gamma k}\right\} .
\end{aligned}
$$

This implies that $\left\{x_{n}\right\}$ is bounded and so are $\left\{z_{n, i}\right\},\left\{h\left(x_{n}\right)\right\}$, $\left\{T\left(t_{n}\right) x_{n}\right\}$, and $\left\{S\left(t_{n}\right) x_{n}\right\}$. Next, we show that $\lim _{n \rightarrow \infty} \| x_{n}-$ $T\left(t_{n}\right) x_{n}\left\|=\lim _{n \rightarrow \infty}\right\| x_{n}-S\left(t_{n}\right) x_{n} \|=0$. Indeed, by Lemma 6 , for each $1 \leq i \leq m$, we have

$$
\begin{aligned}
\left\|z_{n, i}-x^{\star}\right\|^{2} \leq & \left\|x_{n}-x^{\star}\right\|^{2}-\left(1-2 \lambda_{n, i} c_{1, i}\right)\left\|x_{n}-w_{n, i}\right\|^{2} \\
& -\left(1-2 \lambda_{n, i} c_{2, i}\right)\left\|w_{n, i}-z_{n, i}\right\|^{2} .
\end{aligned}
$$

Applying Lemma 4 and inequality (26) for $k \in\{1,2, \ldots, m\}$ we have that

$$
\begin{aligned}
\| y_{n}- & x^{\star} \|^{2} \\
= & \left\|\alpha_{n} x_{n}+\sum_{i=1}^{m} \beta_{n, i} z_{n, i}+\gamma_{n} T\left(t_{n}\right) x_{n}+\eta_{n} S\left(t_{n}\right) x_{n}-x^{\star}\right\|^{2} \\
\leq & \alpha_{n}\left\|x_{n}-x^{\star}\right\|^{2}+\sum_{i=1}^{m} \beta_{n, i}\left\|z_{n, i}-x^{\star}\right\|^{2} \\
& +\gamma_{n}\left\|T\left(t_{n}\right) x_{n}-x^{\star}\right\|^{2}+\eta_{n}\left\|S\left(t_{n}\right) x_{n}-x^{\star}\right\|^{2} \\
& -\alpha_{n} \beta_{n, k}\left\|x_{n}-z_{n, k}\right\|^{2}-\alpha_{n} \gamma_{n}\left\|x_{n}-T\left(t_{n}\right) x_{n}\right\|^{2} \\
& -\alpha_{n} \eta_{n}\left\|x_{n}-S\left(t_{n}\right) x_{n}\right\|^{2}
\end{aligned}
$$




$$
\begin{aligned}
\leq & \alpha_{n}\left\|x_{n}-x^{\star}\right\|^{2}+\sum_{i=1}^{m} \beta_{n, i}\left\|x_{n}-x^{\star}\right\|^{2}+\gamma_{n}\left\|x_{n}-x^{\star}\right\|^{2} \\
& +\eta_{n}\left\|x_{n}-x^{\star}\right\|^{2}-\alpha_{n} \beta_{n, k}\left\|x_{n}-z_{n, k}\right\|^{2} \\
& -\beta_{n, k}\left(1-2 \lambda_{n, k} c_{1, k}\right)\left\|x_{n}-w_{n, k}\right\|^{2} \\
& -\alpha_{n} \gamma_{n}\left\|x_{n}-T\left(t_{n}\right) x_{n}\right\|^{2}-\alpha_{n} \eta_{n}\left\|x_{n}-S\left(t_{n}\right) x_{n}\right\|^{2} \\
= & \left\|x_{n}-x^{\star}\right\|^{2}-\beta_{n, k}\left(1-2 \lambda_{n, k} c_{1, k}\right)\left\|x_{n}-w_{n, k}\right\|^{2} \\
& -\alpha_{n} \beta_{n, k}\left\|x_{n}-z_{n, k}\right\|^{2}-\alpha_{n} \gamma_{n}\left\|x_{n}-T\left(t_{n}\right) x_{n}\right\|^{2} \\
& -\alpha_{n} \eta_{n}\left\|x_{n}-S\left(t_{n}\right) x_{n}\right\|^{2} .
\end{aligned}
$$

We now compute

$$
\begin{aligned}
\| x_{n+1} & -x^{\star} \|^{2} \\
= & \left\|\theta_{n}\left(\gamma h\left(x_{n}\right)-A x^{\star}\right)+\left(I-\theta_{n} A\right)\left(y_{n}-x^{\star}\right)\right\|^{2} \\
\leq & \theta_{n}^{2}\left\|\gamma h\left(x_{n}\right)-A x^{\star}\right\|^{2}+\left(1-\theta_{n} \bar{\gamma}\right)^{2}\left\|y_{n}-x^{\star}\right\|^{2} \\
& +2 \theta_{n}\left(1-\theta_{n} \bar{\gamma}\right)\left\|\gamma h\left(x_{n}\right)-A x^{\star}\right\|\left\|y_{n}-x^{\star}\right\| \\
\leq & \theta_{n}^{2}\left\|\gamma h\left(x_{n}\right)-A x^{\star}\right\|^{2}+\left(1-\theta_{n} \bar{\gamma}\right)^{2}\left\|x_{n}-x^{\star}\right\|^{2} \\
& +2 \theta_{n}\left(1-\theta_{n} \bar{\gamma}\right)\left\|\gamma h\left(x_{n}\right)-A x^{\star}\right\|\left\|x_{n}-x^{\star}\right\| \\
& -\left(1-\theta_{n} \bar{\gamma}\right)^{2} \beta_{n, k}\left(1-2 \lambda_{n, k} c_{1, k}\right)\left\|x_{n}-w_{n, k}\right\|^{2} \\
& -\left(1-\theta_{n} \bar{\gamma}\right)^{2} \alpha_{n} \beta_{n, k}\left\|x_{n}-z_{n, k}\right\|^{2} \\
& -\left(1-\theta_{n} \bar{\gamma}\right)^{2} \alpha_{n} \gamma_{n}\left\|x_{n}-T\left(t_{n}\right) x_{n}\right\|^{2} \\
& -\left(1-\theta_{n} \bar{\gamma}\right)^{2} \alpha_{n} \eta_{n}\left\|x_{n}-S\left(t_{n}\right) x_{n}\right\|^{2} .
\end{aligned}
$$

Therefore,

$$
\begin{aligned}
\left(1-\theta_{n} \bar{\gamma}\right)^{2} \alpha_{n} \beta_{n, k}\left\|x_{n}-z_{n, k}\right\|^{2} \\
\leq\left\|x_{n}-x^{\star}\right\|^{2}-\left\|x_{n+1}-x^{\star}\right\|^{2}+2 \theta_{n}\left(1-\theta_{n} \bar{\gamma}\right) \\
\quad \times\left\|\gamma h\left(x_{n}\right)-A x^{\star}\right\|\left\|x_{n}-x^{\star}\right\|+\theta_{n}^{2}\left\|\gamma h\left(x_{n}\right)-A x^{\star}\right\|^{2} .
\end{aligned}
$$

In order to prove that $x_{n} \rightarrow x^{\star}$ as $n \rightarrow \infty$, we consider the following two cases.

Case 1. Assume that $\left\{\left\|x_{n}-x^{\star}\right\|\right\}$ is a monotone sequence. In other words, for large enough $n_{0},\left\{\left\|x_{n}-x^{\star}\right\|\right\}_{n \geq n_{0}}$ is either nondecreasing or nonincreasing. Since $\left\{\left\|x_{n}-x^{\star}\right\|\right\}$ is bounded, it is convergent. Since $\lim _{n \rightarrow \infty} \theta_{n}=0$ and $\left\{h\left(x_{n}\right)\right\}$ and $\left\{x_{n}\right\}$ are bounded, from (29), we have

$$
\lim _{n \rightarrow \infty}\left(1-\theta_{n} \bar{\gamma}\right)^{2} \alpha_{n} \beta_{n, k}\left\|x_{n}-z_{n, k}\right\|^{2}=0
$$

and by assumption we get

$$
\lim _{n \rightarrow \infty}\left\|x_{n}-z_{n, k}\right\|=0, \quad 1 \leq k \leq m .
$$

By similar argument we can obtain that

$$
\begin{aligned}
\lim _{n \rightarrow \infty}\left\|x_{n}-w_{n, k}\right\| & =\lim _{n \rightarrow \infty}\left\|x_{n}-T\left(t_{n}\right) x_{n}\right\| \\
& =\lim _{n \rightarrow \infty}\left\|x_{n}-S\left(t_{n}\right) x_{n}\right\|=0 .
\end{aligned}
$$

Further, for all $h \geq 0$ and $n \geq 0$, we see that

$$
\begin{aligned}
&\left\|x_{n}-T(h) x_{n}\right\| \\
& \leq\left\|x_{n}-T\left(t_{n}\right) x_{n}\right\|+\left\|T\left(t_{n}\right) x_{n}-T(h) T\left(t_{n}\right) x_{n}\right\| \\
&+\left\|T(h) T\left(t_{n}\right) x_{n}-T(h) x_{n}\right\| \\
& \leq 2\left\|x_{n}-T\left(t_{n}\right) x_{n}\right\| \\
&+\sup _{x \in\left\{x_{n}\right\}}\left\|T\left(t_{n}\right) x_{n}-T(h) T\left(t_{n}\right) x_{n}\right\| .
\end{aligned}
$$

Since $\{T(t)\}$ is u.a.r. nonexpansive semigroup and $\lim _{n \rightarrow \infty} t_{n}=\infty$, we have

$$
\lim _{n \rightarrow \infty}\left\|x_{n}-T(h) x_{n}\right\|=0 .
$$

Similarly, for all $h \geq 0$ and $n \geq 0$, we obtain that

$$
\lim _{n \rightarrow \infty}\left\|x_{n}-S(h) x_{n}\right\|=0
$$

Next, we show that

$$
\limsup _{n \rightarrow \infty}\left\langle(A-\gamma h) x^{\star}, x^{\star}-x_{n}\right\rangle \leq 0 .
$$

To show this inequality, we choose a subsequence $\left\{x_{n_{i}}\right\}$ of $\left\{x_{n}\right\}$ such that

$$
\begin{aligned}
& \lim _{i \rightarrow \infty}\left\langle(A-\gamma h) x^{\star}, x^{\star}-x_{n_{i}}\right\rangle \\
& \quad=\limsup _{n \rightarrow \infty}\left\langle(A-\gamma h) x^{\star}, x^{\star}-x_{n}\right\rangle .
\end{aligned}
$$

Since $\left\{x_{n_{i}}\right\}$ is bounded, there exists a subsequence $\left\{x_{n_{i_{j}}}\right\}$ of $\left\{x_{n_{i}}\right\}$ which converges weakly to $\tilde{x}$. Without loss of generality, we can assume that $x_{n_{j}} \rightarrow \tilde{x}$. Consider

$$
\begin{aligned}
\left\|x_{n_{j}}-T(t) \tilde{x}\right\| & \leq\left\|x_{n_{j}}-T(t) x_{n_{j}}\right\|+\left\|T(t) x_{n_{j}}-T(t) \tilde{x}\right\| \\
& \leq\left\|x_{n_{j}}-T(t) x_{n_{j}}\right\|+\left\|x_{n_{j}}-\tilde{x}\right\| .
\end{aligned}
$$

Thus, we have

$$
\limsup _{n \rightarrow \infty}\left\|x_{n_{i}}-T(t) \tilde{x}\right\| \leq \limsup _{n \rightarrow \infty}\left\|x_{n_{i}}-\tilde{x}\right\| .
$$

By the Opial property of the Hilbert space $H$ we obtain $T(t) \tilde{x}=\tilde{x}$ for all $t \geq 0$. Similarly we have that $S(t) \tilde{x}=\tilde{x}$ for all $t \geq 0$. This implies that $\tilde{x} \in F(\mathscr{T}) \bigcap F(\mathcal{S})$. Now we show that $\tilde{x} \in \operatorname{Sol}\left(f_{i}, C\right)$. For each $1 \leq i \leq m$, since $f_{i}(x, \cdot)$ is convex on $C$ for each $x \in C$, we see that

$$
w_{n, i}=\operatorname{argmin}\left\{\lambda_{n, i} f_{i}\left(x_{n}, y\right)+\frac{1}{2}\left\|y-x_{n}\right\|^{2}: y \in C\right\}
$$


if and only if

$$
0 \in \partial_{2}\left(f_{i}\left(x_{n}, y\right)+\frac{1}{2}\left\|y-x_{n}\right\|^{2}\right)\left(w_{n, i}\right)+N_{C}\left(w_{n, i}\right)
$$

where $N_{C}(x)$ is the (outward) normal cone of $C$ at $x \in C$. This follows that

$$
0=\lambda_{n, i} v+w_{n, i}-x_{n}+u_{n}
$$

where $v \in \partial_{2} f_{i}\left(x_{n}, w_{n, i}\right)$ and $u_{n} \in N_{C}\left(w_{n, i}\right)$. By the definition of the normal cone $N_{C}$ we have

$$
\left\langle w_{n, i}-x_{n}, y-w_{n, i}\right\rangle \geq \lambda_{n, i}\left\langle v, w_{n, i}-y\right\rangle, \quad \forall y \in C .
$$

Since $f_{i}\left(x_{n}, \cdot\right)$ is subdifferentiable on $C$, by the well-known Moreau-Rockafellar theorem [31] (also see [6]), for $v \in$ $\partial_{2} f_{i}\left(x_{n}, w_{n, i}\right)$, we have

$$
f_{i}\left(x_{n}, y\right)-f_{i}\left(x_{n}, w_{n, i}\right) \geq\left\langle v, y-w_{n, i}\right\rangle, \quad \forall y \in C .
$$

Combining this with (43), we have

$$
\begin{array}{r}
\lambda_{n, i}\left(f_{i}\left(x_{n}, y\right)-f_{i}\left(x_{n}, w_{n, i}\right)\right) \geq\left\langle w_{n, i}-x_{n}, w_{n, i}-y\right\rangle, \\
\forall y \in C .
\end{array}
$$

In particular, we have

$$
\lambda_{n_{j}, i}\left(f_{i}\left(x_{n_{j}}, y\right)-f_{i}\left(x_{n_{j}}, w_{n, i}\right)\right) \geq\left\langle w_{n_{j}, i}-x_{n_{j}}, w_{n_{j}, i}-y\right\rangle
$$

Since $x_{n_{j}} \rightarrow \tilde{x}$, it follows from (32) that $w_{n_{j}, i} \rightarrow \tilde{x}$. And thus we have

$$
f_{i}(\tilde{x}, y) \geq 0, \quad \forall y \in C
$$

This implies that $\tilde{x} \in \operatorname{Sol}\left(f_{i}, C\right)$ and hence $\tilde{x} \in \Omega$. Since $x^{\star}=$ $\operatorname{Proj}_{\Omega}(I-A+\gamma h) x^{\star}$ and $\tilde{x} \in \Omega$, we have

$$
\begin{gathered}
\limsup _{n \rightarrow \infty}\left\langle(A-\gamma h) x^{\star}, x^{\star}-x_{n}\right\rangle \\
\quad=\lim _{i \rightarrow \infty}\left\langle(A-\gamma h) x^{\star}, x^{\star}-x_{n_{i}}\right\rangle \\
\quad=\left\langle(A-\gamma h) x^{\star}, x^{\star}-\tilde{x}\right\rangle \leq 0 .
\end{gathered}
$$

From Lemma 1, it follows that

$$
\begin{aligned}
\| x_{n+1} & -x^{\star} \|^{2} \\
\leq & \left\|\left(I-\theta_{n} A\right)\left(y_{n}-x^{\star}\right)\right\|^{2} \\
& +2 \theta_{n}\left\langle\gamma h\left(x_{n}\right)-A x^{\star}, x_{n+1}-x^{\star}\right\rangle \\
\leq & \left(1-\theta_{n} \bar{\gamma}\right)^{2}\left\|x_{n}-x^{\star}\right\|^{2} \\
& +2 \theta_{n} \gamma\left\langle h\left(x_{n}\right)-h\left(x^{\star}\right), x_{n+1}-x^{\star}\right\rangle \\
& +2 \theta_{n}\left\langle\gamma h\left(x^{\star}\right)-A x^{\star}, x_{n+1}-x^{\star}\right\rangle \\
\leq & \left(1-\theta_{n} \bar{\gamma}\right)^{2}\left\|x_{n}-x^{\star}\right\|^{2}+2 \theta_{n} k \gamma\left\|x_{n}-x^{\star}\right\|\left\|x_{n+1}-x^{\star}\right\| \\
& +2 \theta_{n}\left\langle\gamma h\left(x^{\star}\right)-A x^{\star}, x_{n+1}-x^{\star}\right\rangle \\
\leq & \left(1-\theta_{n} \bar{\gamma}\right)^{2}\left\|x_{n}-x^{\star}\right\|^{2} \\
& +\theta_{n} k \gamma\left(\left\|x_{n}-x^{\star}\right\|^{2}+\left\|x_{n+1}-x^{\star}\right\|^{2}\right) \\
& +2 \theta_{n}\left\langle\gamma h\left(x^{\star}\right)-A x^{\star}, x_{n+1}-x^{\star}\right\rangle \\
\leq & \left(\left(1-\theta_{n} \bar{\gamma}\right)^{2}+\theta_{n} k \gamma\right)\left\|x_{n}-x^{\star}\right\|^{2} \\
& +\theta_{n} \gamma k\left\|x_{n+1}-x^{\star}\right\|^{2}+2 \theta_{n}\left\langle\gamma h\left(x^{\star}\right)-A x^{\star}, x_{n+1}-x^{\star}\right\rangle .
\end{aligned}
$$

This implies that

$$
\begin{aligned}
& \left\|x_{n+1}-x^{\star}\right\|^{2} \\
& \leq \frac{1-2 \theta_{n} \bar{\gamma}+\left(\theta_{n} \bar{\gamma}\right)^{2}+\theta_{n} \gamma k}{1-\theta_{n} \gamma k}\left\|x_{n}-x^{\star}\right\|^{2} \\
& +\frac{2 \theta_{n}}{1-\theta_{n} \gamma k}\left\langle\gamma h\left(x^{\star}\right)-A x^{\star}, x_{n+1}-x^{\star}\right\rangle \\
& =\left(1-\frac{2(\bar{\gamma}-\gamma k) \theta_{n}}{1-\theta_{n} \gamma k}\right)\left\|x_{n}-x^{\star}\right\|^{2}+\frac{\left(\theta_{n} \bar{\gamma}\right)^{2}}{1-\theta_{n} \gamma k}\left\|x_{n}-x^{\star}\right\|^{2} \\
& +\frac{2 \theta_{n}}{1-\theta_{n} \gamma k}\left\langle\gamma h\left(x^{\star}\right)-A x^{\star}, x_{n+1}-x^{\star}\right\rangle \\
& \leq\left(1-\frac{2(\bar{\gamma}-\gamma k) \theta_{n}}{1-\theta_{n} \gamma k}\right)\left\|x_{n}-x^{\star}\right\|^{2}+\frac{2(\bar{\gamma}-\gamma k) \theta_{n}}{1-\theta_{n} \gamma k} \\
& \times\left(\frac{\left(\theta_{n} \bar{\gamma}^{2}\right) M}{2(\bar{\gamma}-\gamma k)}+\frac{1}{\bar{\gamma}-\gamma k}\left\langle\gamma h\left(x^{\star}\right)-A x^{\star}, x_{n+1}-x^{\star}\right\rangle\right) \\
& =\left(1-\eta_{n}\right)\left\|x_{n}-x^{\star}\right\|^{2}+\eta_{n} \delta_{n},
\end{aligned}
$$


where

$$
\begin{gathered}
M=\sup \left\{\left\|x_{n}-x^{\star}\right\|^{2}: n \geq 0\right\}, \quad \eta_{n}=\frac{2(\bar{\gamma}-\gamma k) \theta_{n}}{1-\theta_{n} \gamma k}, \\
\delta_{n}=\frac{\left(\theta_{n} \bar{\gamma}^{2}\right) M}{2(\bar{\gamma}-\gamma k)}+\frac{1}{\bar{\gamma}-\gamma k}\left\langle\gamma h x^{\star}-A x^{\star}, x_{n+1}-x^{\star}\right\rangle .
\end{gathered}
$$

It is easy to see that $\eta_{n} \rightarrow 0, \sum_{n=1}^{\infty} \eta_{n}=\infty$, and $\lim \sup _{n \rightarrow \infty} \delta_{n} \leq 0$. Hence, by Lemma 2 the sequence $\left\{x_{n}\right\}$ converges strongly to $x^{\star}$. From (32) we have that $\left\{w_{n, i}\right\}$ and $\left\{z_{n, i}\right\}$ converge strongly to $x^{\star}$.

Case 2. Assume that $\left\{\left\|x_{n}-x^{\star}\right\|\right\}$ is not a monotone sequence. Then, we can define an integer sequence $\{\tau(n)\}$ for all $n \geq n_{0}$ (for some large enough $n_{0}$ ) by

$$
\tau(n):=\max \left\{k \in \mathbb{N}, k \leq n:\left\|x_{k}-x^{\star}\right\|<\left\|x_{k+1}-x^{\star}\right\|\right\} .
$$

Clearly, $\tau$ is a nondecreasing sequence such that $\tau(n) \rightarrow \infty$ as $n \rightarrow \infty$, and for all $n \geq n_{0}$,

$$
\left\|x_{\tau(n)}-x^{\star}\right\|<\left\|x_{\tau(n)+1}-x^{\star}\right\| .
$$

From (33) we obtain that

$$
\begin{aligned}
\lim _{n \rightarrow \infty}\left\|x_{\tau(n)}-z_{\tau(n), i}\right\| & =\lim _{n \rightarrow \infty}\left\|x_{\tau(n)}-w_{\tau(n), i}\right\| \\
& =\lim _{n \rightarrow \infty}\left\|x_{\tau(n)}-T\left(t_{\tau_{n}}\right) x_{\tau(n)}\right\|=0 .
\end{aligned}
$$

Following an argument similar to that in Case 1 we have

$$
\left\|x_{\tau(n)+1}-x^{\star}\right\|^{2} \leq\left(1-\eta_{\tau(n)}\right)\left\|x_{\tau(n)}-x^{\star}\right\|^{2}+\eta_{\tau(n)} \delta_{\tau(n)},
$$

where $\eta_{\tau(n)} \rightarrow 0, \sum_{n=1}^{\infty} \eta_{\tau(n)}=\infty$, and $\lim \sup _{n \rightarrow \infty} \delta_{\tau(n)} \leq 0$. Hence, by Lemma 2, we obtain $\lim _{n \rightarrow \infty}\left\|x_{\tau(n)}-x^{\star}\right\|=0$ and $\lim _{n \rightarrow \infty}\left\|x_{\tau(n)+1}-x^{\star}\right\|=0$. Now Lemma 5 implies that

$$
\begin{aligned}
0 & \leq\left\|x_{n}-x^{\star}\right\| \leq \max \left\{\left\|x_{\tau(n)}-x^{\star}\right\|,\left\|x_{n}-x^{\star}\right\|\right\} \\
& \leq\left\|x_{\tau(n)+1}-x^{\star}\right\| .
\end{aligned}
$$

Therefore, $\left\{x_{n}\right\}$ converges strongly to $x^{\star}=\operatorname{Proj}_{\Omega}(I-A+$ $\gamma h) x^{\star}$. This completes the proof.

\section{Application}

In this section, we consider a particular Fan inequality corresponding to the function $f$ defined by the following: for every $x, y \in C$

$$
f(x, y)=\langle F(x), y-x\rangle
$$

where $F: C \rightarrow H$. Then, we obtain the classical variational inequality as follows.

Find $z \in C$ such that $\langle F(z), y-z\rangle \geq 0, \forall y \in C$.
The set of solutions of this problem is denoted by $\operatorname{VI}(F, C)$. In that particular case, the solution $y_{n}$ of the minimization problem

$$
\operatorname{argmin}\left\{\lambda_{n} f\left(x_{n}, y\right)+\frac{1}{2}\left\|y-x_{n}\right\|^{2}: y \in C\right\}
$$

can be expressed as

$$
y_{n}=P_{C}\left(x_{n}-\lambda_{n} F\left(x_{n}\right)\right) .
$$

Let $F$ be $L$-Lipschitz continuous on $C$. Then

$$
\begin{array}{r}
f(x, y)+f(y, z)-f(x, z)=\langle F(x)-F(y), y-z\rangle, \\
x, y, z \in C .
\end{array}
$$

Therefore,

$$
\begin{aligned}
|\langle F(x)-F(y), y-z\rangle| & \leq L\|x-y\|\|y-z\| \\
& \leq \frac{L}{2}\left(\|x-y\|^{2}+\|y-z\|^{2}\right)
\end{aligned}
$$

hence, $f$ satisfies Lipschitz-type continuous condition with $c_{1}=c_{2}=L / 2$.

Using Theorem 7 we obtain the following convergence theorem.

Theorem 8. Let $C$ be a nonempty closed convex subset of a real Hilbert space $H$ and let $F_{i}: C \rightarrow H(i=$ $1,2, \ldots, m)$ be functions such that, for each $1 \leq i \leq m$, $F_{i}$ is monotone and L-Lipschitz continuous on C. Let $\mathscr{T}=$ $\{T(u): u \geq 0\}$ and $\mathcal{S}=\{S(u): u \geq 0\}$ be two u.a.r. nonexpansive self-mapping semigroups on $C$ such that $\Omega=\bigcap_{i=1}^{m} V I\left(F_{i}, C\right) \bigcap F(\mathscr{T}) \bigcap F(\mathcal{S}) \neq \emptyset$. Assume that $h$ is a $k$ contraction of $C$ into itself and $A$ is a strongly positive bounded linear self-adjoint operator on $H$ with coefficient $\bar{\gamma}<1$ and $0<\gamma<\bar{\gamma} / k$. Let $x_{0} \in C$ and let $\left\{x_{n}\right\},\left\{w_{n, i}\right\}$, and $\left\{z_{n, i}\right\}$ be sequences generated by

$$
\begin{gathered}
w_{n, i}=P_{C}\left(x_{n}-\lambda_{n, i} F_{i}\left(x_{n}\right)\right), \quad i \in\{1,2, \ldots, m\}, \\
z_{n, i}=P_{C}\left(x_{n}-\lambda_{n, i} F_{i}\left(w_{n, i}\right)\right), \quad i \in\{1,2, \ldots, m\}, \\
y_{n}=\alpha_{n} x_{n}+\sum_{i=1}^{m} \beta_{n, i} z_{n, i}+\gamma_{n} T\left(t_{n}\right) x_{n}+\eta_{n} S\left(t_{n}\right) x_{n}, \\
x_{n+1}=\theta_{n} \gamma h\left(x_{n}\right)+\left(I-\theta_{n} A\right) y_{n}, \quad \forall n \geq 0,
\end{gathered}
$$

where $\alpha_{n}+\sum_{i=1}^{m} \beta_{n, i}+\gamma_{n}+\eta_{n}=1$ and $\left\{\alpha_{n}\right\},\left\{\beta_{n, i}\right\},\left\{\gamma_{n}\right\},\left\{\eta_{n}\right\}$, $\left\{\lambda_{n, i}\right\}$, and $\left\{\theta_{n}\right\}$ satisfy the following conditions:

(i) $\lim _{n \rightarrow \infty} t_{n}=\infty$,

(ii) $\left.\left\{\theta_{n}\right\} \subset\right] 0,1\left[, \lim _{n \rightarrow \infty} \theta_{n}=0\right.$, and $\sum_{n=1}^{\infty} \theta_{n}=\infty$,

(iii) $\left.\left\{\lambda_{n, i}\right\} \subset[a, b] \subset\right] 0,1 / L[$,

(iv) $\left.\left\{\alpha_{n}\right\},\left\{\beta_{n, i}\right\},\left\{\gamma_{n}\right\},\left\{\eta_{n}\right\} \subset\right] 0,1\left[, \liminf _{n} \alpha_{n} \beta_{n, i}>0\right.$, $\liminf _{n} \alpha_{n} \gamma_{n}>0, \liminf \alpha_{n} \eta_{n}>0$, and $\liminf _{n} \beta_{n, i}\left(1-2 \lambda_{n, i} c_{1, i}\right)>0$ for each $1 \leq i \leq m$.

Then, the sequence $\left\{x_{n}\right\}$ converges strongly to $x^{\star} \in$ $\bigcap_{i=1}^{m} V I\left(F_{i}, C\right) \bigcap F(\mathscr{T}) \bigcap F(\mathcal{S})$ which solves the variational inequality

$$
\left\langle(A-\gamma h) x^{\star}, x-x^{\star}\right\rangle \geq 0, \quad \forall x \in \Omega .
$$


In [32], Baillon proved a strong mean convergence theorem for nonexpansive mappings, and it was generalized in [33]. It follows from the above proof that Theorems 7 is valid for nonexpansive mappings. Thus, we have the following mean ergodic theorems for nonexpansive mappings in a Hilbert space.

Theorem 9. Let $C$ be a nonempty closed convex subset of a real Hilbert space $H$ and let $F_{i}: C \rightarrow H(i=$ $1,2, \ldots, m)$ be functions such that for each $1 \leq i \leq$ $m, F_{i}$ is monotone and L-Lipschitz continuous on C. Let $T$ and $S$ be two nonexpansive mappings on $C$ such that $\Omega=\bigcap_{i=1}^{m} V I\left(F_{i}, C\right) \bigcap F(T) \bigcap F(S) \neq \emptyset$. Assume that $h$ is a $k$ contraction of $C$ into itself and $A$ is a strongly positive bounded linear self-adjoint operator on $H$ with coefficient $\bar{\gamma}<1$ and $0<\gamma<\bar{\gamma} / k$. Let $\left\{x_{n}\right\},\left\{w_{n, i}\right\}$, and $\left\{z_{n, i}\right\}$ be sequences generated by $x_{0} \in C$ and by

$$
\begin{gathered}
w_{n, i}=P_{C}\left(x_{n}-\lambda_{n, i} F_{i}\left(x_{n}\right)\right), \quad i \in\{1,2, \ldots, m\}, \\
z_{n, i}=P_{C}\left(x_{n}-\lambda_{n, i} F_{i}\left(w_{n, i}\right)\right), \quad i \in\{1,2, \ldots, m\}, \\
y_{n}=\alpha_{n} x_{n}+\sum_{i=1}^{m} \beta_{n, i} z_{n, i}+\gamma_{n} T^{n} x_{n}+\eta_{n} S^{n} x_{n}, \\
x_{n+1}=\theta_{n} \gamma h\left(x_{n}\right)+\left(I-\theta_{n} A\right) y_{n}, \quad \forall n \geq 0,
\end{gathered}
$$

where $\alpha_{n}+\sum_{i=1}^{m} \beta_{n, i}+\gamma_{n}+\eta_{n}=1$ and $\left\{\alpha_{n}\right\},\left\{\beta_{n, i}\right\},\left\{\gamma_{n}\right\},\left\{\eta_{n}\right\}$, $\left\{\lambda_{n, i}\right\}$, and $\left\{\theta_{n}\right\}$ satisfy the following conditions:

(i) $\left.\left\{\theta_{n}\right\} \subset\right] 0,1\left[, \lim _{n \rightarrow \infty} \theta_{n}=0\right.$, and $\sum_{n=1}^{\infty} \theta_{n}=\infty$,

(ii) $\left.\left\{\lambda_{n, i}\right\} \subset[a, b] \subset\right] 0,1 / L\left[\right.$, where $L=\max \left\{2 c_{1, i}, 2 c_{2, i}\right.$, $1 \leq i \leq m\}$,

(iii) $\left.\left\{\alpha_{n}\right\},\left\{\beta_{n, i}\right\},\left\{\gamma_{n}\right\},\left\{\eta_{n}\right\} \quad \subset\right] 0,1\left[, \liminf _{n} \alpha_{n} \beta_{n, i}>0\right.$, $\liminf _{n} \alpha_{n} \gamma_{n}>0, \liminf _{n} \alpha_{n} \eta_{n}>0$, and $\lim \inf _{n} \beta_{n, i}\left(1-2 \lambda_{n, i} c_{1, i}\right)>0$ for each $1 \leq i \leq m$.

Then, the sequence $\left\{x_{n}\right\}$ converges strongly to $x^{\star} \in$ $\bigcap_{i=1}^{m} \operatorname{VI}\left(F_{i}, C\right) \bigcap F(\mathscr{T}) \bigcap F(\mathcal{S})$ which solves the variational inequality

$$
\left\langle(A-\gamma h) x^{\star}, x-x^{\star}\right\rangle \geq 0, \quad \forall x \in \Omega .
$$

\section{Conflict of Interests}

The authors declare that there is no conflict of interests regarding the publication of this paper.

\section{Acknowledgment}

This paper was funded by the Deanship of Scientific Research (DSR), King Abdulaziz University, Jeddah. Therefore, the authors acknowledge with thanks DSR, for technical and financial support.

\section{References}

[1] K. Fan, "A minimax inequality and applications," in Inequality III, pp. 103-113, Academic Press, New York, NY, USA, 1972.
[2] P. N. Anh, "A hybrid extragradient method extended to fixed point problems and equilibrium problems," Optimization, vol. 62, no. 2, pp. 271-283, 2013.

[3] E. Blum and W. Oettli, "From optimization and variational inequalities to equilibrium problems," The Mathematics Student, vol. 63, no. 1-4, pp. 123-145, 1994.

[4] L.-C. Ceng, N. Hadjisavvas, and N.-C. Wong, "Strong convergence theorem by a hybrid extragradient-like approximation method for variational inequalities and fixed point problems," Journal of Global Optimization, vol. 46, no. 4, pp. 635-646, 2010.

[5] G. Stampacchia, "Formes bilinéaires coercitives sur les ensembles convexes," Comptes rendus de l'Académie des Sciences, vol. 258, pp. 4413-4416, 1964.

[6] P. Daniele, F. Giannessi, and A. Maugeri, Equilibrium Problems and Variational Models, vol. 68, Kluwer Academic, Norwell, Mass, USA, 2003.

[7] J.-W. Peng and J.-C. Yao, "Some new extragradient-like methods for generalized equilibrium problems, fixed point problems and variational inequality problems," Optimization Methods \& Software, vol. 25, no. 4-6, pp. 677-698, 2010.

[8] D. R. Sahu, N.-C. Wong, and J.-C. Yao, "Strong convergence theorems for semigroups of asymptotically nonexpansive mappings in Banach spaces," Abstract and Applied Analysis, vol. 2013, Article ID 202095, 8 pages, 2013.

[9] S. Takahashi and W. Takahashi, "Viscosity approximation methods for equilibrium problems and fixed point problems in Hilbert spaces," Journal of Mathematical Analysis and Applications, vol. 331, no. 1, pp. 506-515, 2007.

[10] S. Wang and B. Guo, "New iterative scheme with nonexpansive mappings for equilibrium problems and variational inequality problems in Hilbert spaces," Journal of Computational and Applied Mathematics, vol. 233, no. 10, pp. 2620-2630, 2010.

[11] Y. Yao, Y.-C. Liou, and Y.-J. Wu, "An extragradient method for mixed equilibrium problems and fixed point problems," Fixed Point Theory and Applications, vol. 2009, Article ID 632819, 15 pages, 2009.

[12] F. E. Browder, "Nonexpansive nonlinear operators in a Banach space," Proceedings of the National Academy of Sciences of the United States of America, vol. 54, pp. 1041-1044, 1965.

[13] R. Chen and Y. Song, "Convergence to common fixed point of nonexpansive semigroups," Journal of Computational and Applied Mathematics, vol. 200, no. 2, pp. 566-575, 2007.

[14] A. Aleyner and Y. Censor, "Best approximation to common fixed points of a semigroup of nonexpansive operators," Journal of Nonlinear and Convex Analysis, vol. 6, no. 1, pp. 137-151, 2005.

[15] H. K. Xu, "An iterative approach to quadratic optimization," Journal of Optimization Theory and Applications, vol. 116, no. 3, pp. 659-678, 2003.

[16] G. Marino and H.-K. Xu, "A general iterative method for nonexpansive mappings in Hilbert spaces," Journal of Mathematical Analysis and Applications, vol. 318, no. 1, pp. 43-52, 2006.

[17] A. Moudafi, "Viscosity approximation methods for fixed-points problems," Journal of Mathematical Analysis and Applications, vol. 241, no. 1, pp. 46-55, 2000.

[18] F. Cianciaruso, G. Marino, and L. Muglia, "Iterative methods for equilibrium and fixed point problems for nonexpansive semigroups in Hilbert spaces," Journal of Optimization Theory and Applications, vol. 146, no. 2, pp. 491-509, 2010.

[19] S. Li, L. Li, and Y. Su, "General iterative methods for a oneparameter nonexpansive semigroup in Hilbert space," Nonlinear Analysis: Theory, Methods \& Applications, vol. 70, no. 9, pp. 3065-3071, 2009. 
[20] D. R. Sahu, N. C. Wong, and J. C. Yao, "A unified hybrid iterative method for solving variational inequalities involving generalized pseudocontractive mappings," SIAM Journal on Control and Optimization, vol. 50, no. 4, pp. 2335-2354, 2012.

[21] N. Shioji and W. Takahashi, "Strong convergence theorems for asymptotically nonexpansive semigroups in Hilbert spaces," Nonlinear Analysis: Theory, Methods \& Applications, vol. 34, no. 1, pp. 87-99, 1998.

[22] P. N. Anh, "Strong convergence theorems for nonexpansive mappings and Ky Fan inequalities," Journal of Optimization Theory and Applications, vol. 154, no. 1, pp. 303-320, 2012.

[23] L.-C. Ceng, Q. H. Ansari, and J.-C. Yao, "Hybrid proximaltype and hybrid shrinking projection algorithms for equilibrium problems, maximal monotone operators, and relatively nonexpansive mappings," Numerical Functional Analysis and Optimization, vol. 31, no. 7-9, pp. 763-797, 2010.

[24] L.-C. Ceng, Q. H. Ansari, and J.-C. Yao, "Viscosity approximation methods for generalized equilibrium problems and fixed point problems," Journal of Global Optimization, vol. 43, no. 4, pp. 487-502, 2009.

[25] L.-C. Ceng, Q. H. Ansari, and J.-C. Yao, "Relaxed extragradient iterative methods for variational inequalities," Applied Mathematics and Computation, vol. 218, no. 3, pp. 1112-1123, 2011.

[26] L.-C. Ceng, S.-M. Guu, and J.-C. Yao, "Hybrid iterative method for finding common solutions of generalized mixed equilibrium and fixed point problems," Fixed Point Theory and Applications, vol. 2012, article 92, 2012.

[27] M. Eslamian, "Convergence theorems for nonspreading mappings and nonexpansive multivalued mappings and equilibrium problems," Optimization Letters, vol. 7, no. 3, pp. 547-557, 2013.

[28] M. Eslamian, "Hybrid method for equilibrium problems and fixed point problems of finite families of nonexpansive semigroups," RACSAM, vol. 107, pp. 299-307, 2013.

[29] M. Eslamian and A. Abkar, "One-step iterative process for a finite family of multivalued mappings," Mathematical and Computer Modelling, vol. 54, no. 1-2, pp. 105-111, 2011.

[30] P.-E. Maingé, "Strong convergence of projected subgradient methods for nonsmooth and nonstrictly convex minimization," Set-Valued Analysis, vol. 16, no. 7-8, pp. 899-912, 2008.

[31] R. T. Rockafellar, "Monotone operators and the proximal point algorithm," SIAM Journal on Control and Optimization, vol. 14, no. 5, pp. 877-898, 1976.

[32] J.-B. Baillon, "Un théorème de type ergodique pour les contractions non linéaires dans un espace de Hilbert," Comptes Rendus de l'Académie des Sciences, vol. 280, no. 22, pp. A1511-A1514, 1975.

[33] W. Kaczor, T. Kuczumow, and S. Reich, "A mean ergodic theorem for nonlinear semigroups which are asymptotically nonexpansive in the intermediate sense," Journal of Mathematical Analysis and Applications, vol. 246, no. 1, pp. 1-27, 2000. 


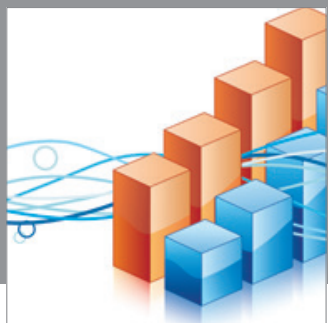

Advances in

Operations Research

mansans

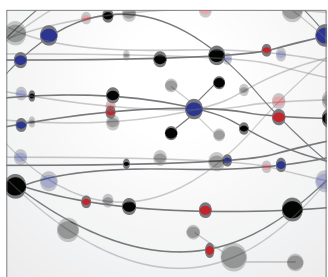

The Scientific World Journal
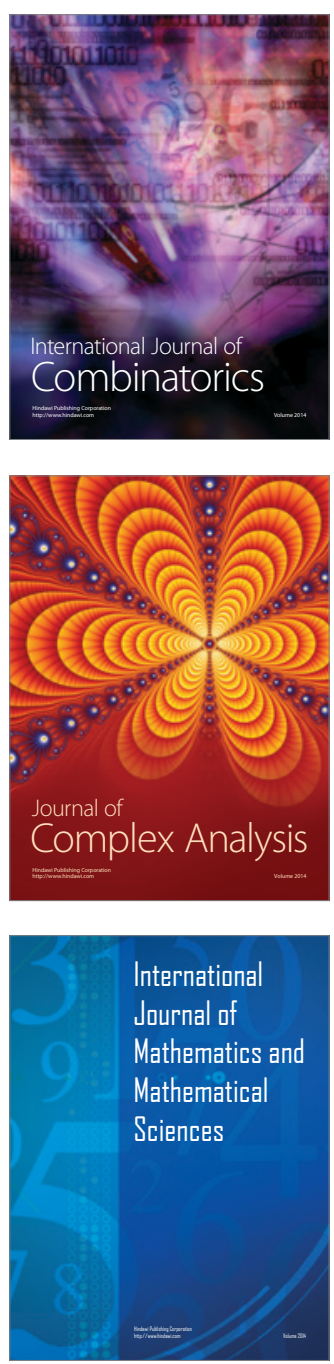
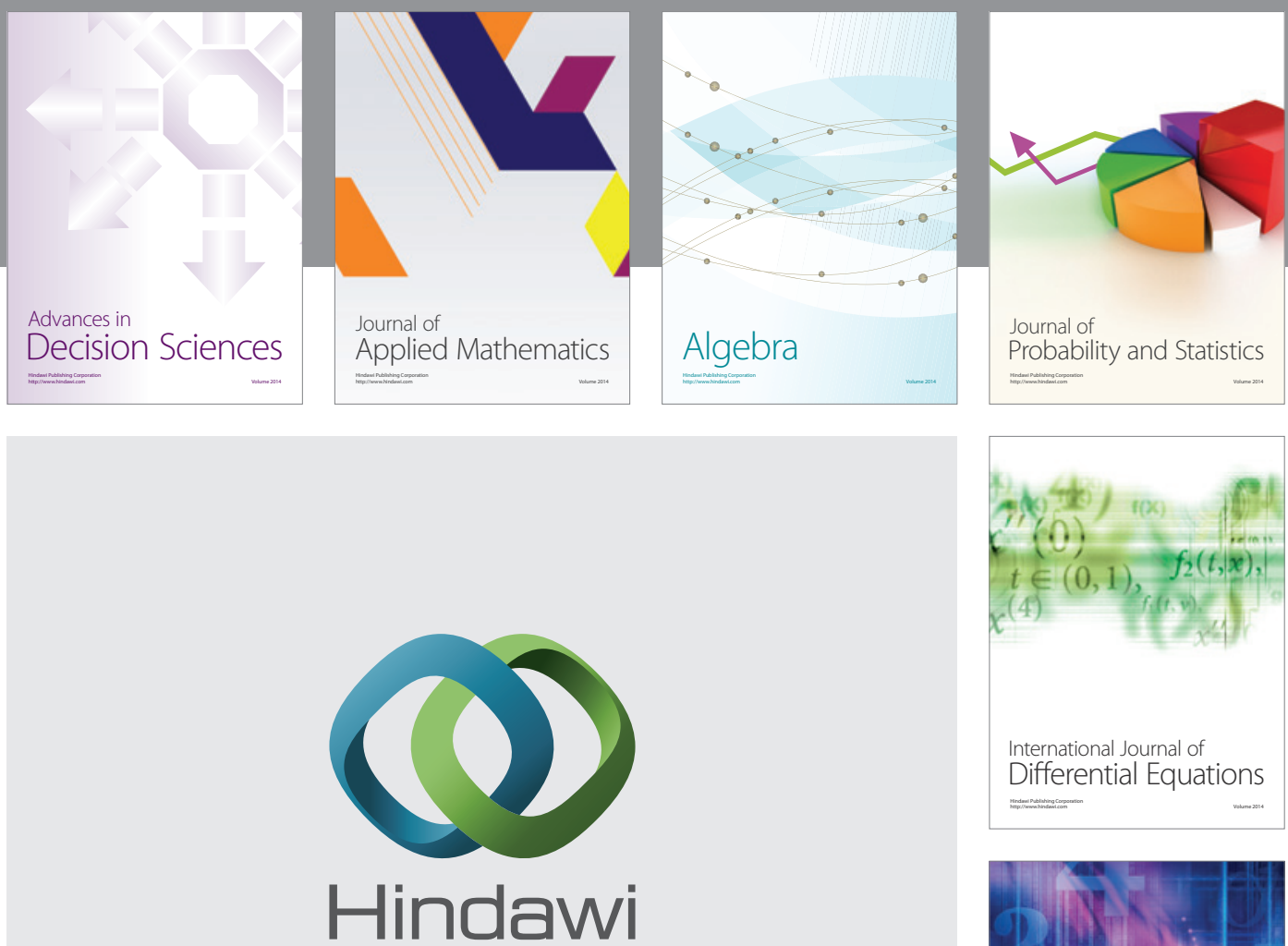

Submit your manuscripts at http://www.hindawi.com
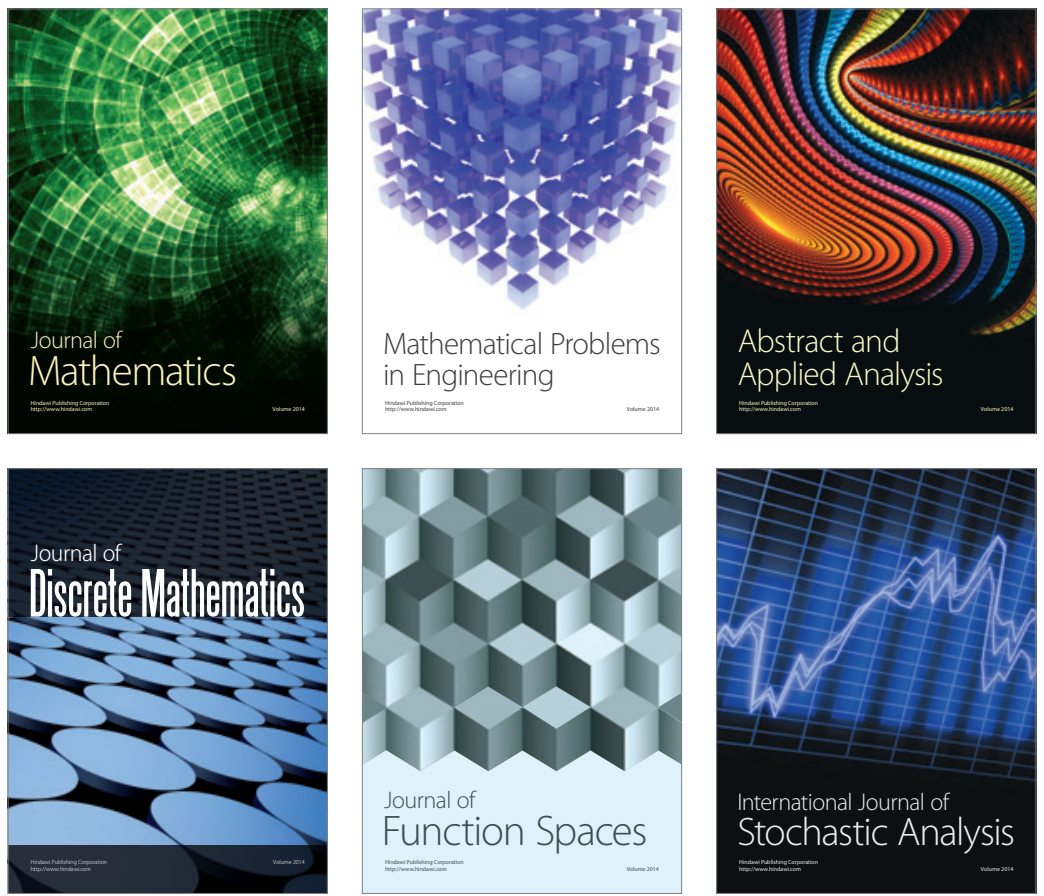

Journal of

Function Spaces

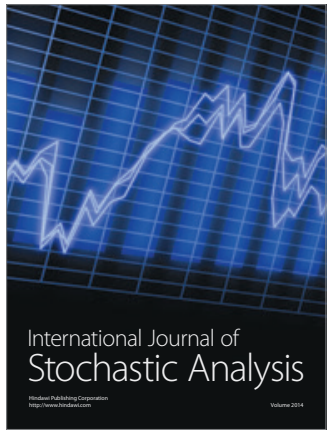

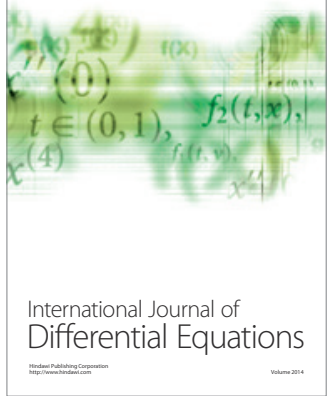
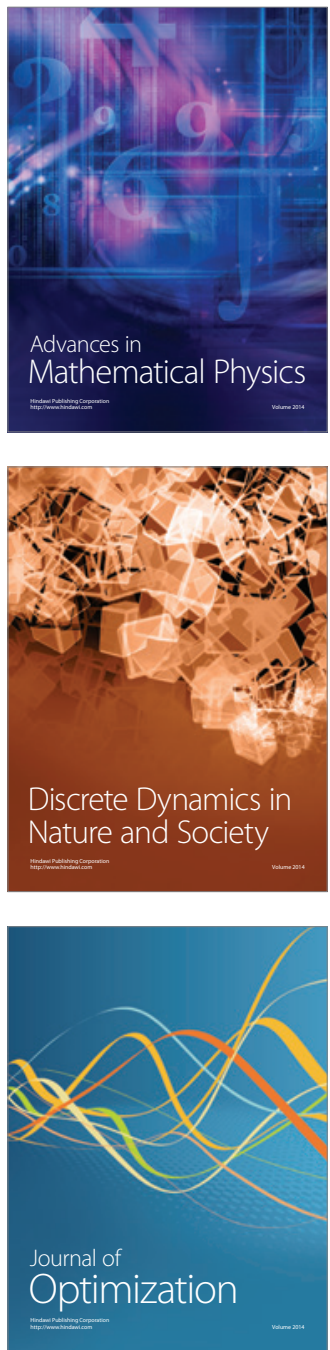